\title{
Prevalence of preoperative alcohol abuse among patients seeking weight-loss surgery
}

\author{
Omar Yusef Kudsi ${ }^{1}$, Karen Huskey², Shannon Grove ${ }^{2}$, George Blackburn ${ }^{3}$, Daniel B. \\ Jones $^{1}$, and Christina C. Wee ${ }^{2,4}$ \\ ${ }^{1}$ Division of Minimally Invasive Surgery, Department of Surgery, Beth Israel Deaconess Medical \\ Center, Harvard Medical School, Boston, MA 02215, USA \\ 2 Division of General Medicine and Primary Care, Department of Medicine, Beth Israel \\ Deaconess Medical Center, Harvard Medical School, Boston, MA 02215, USA
}

${ }^{3}$ Center for the Study of Nutrition, Beth Israel Deaconess Medical Center, Harvard Medical School, Boston, MA 02215, USA

${ }^{4}$ Division of General Medicine and Primary Care, Beth Israel Deaconess Medical Center, 330 Brookline Ave, Boston, MA 02215, USA

\begin{abstract}
Background-It has been hypothesized that alcohol metabolism is altered after weight-loss surgery (WLS), with a few studies suggesting a high prevalence of postoperative problem drinking. However, many of these studies were methodologically limited by lack of preoperative alcohol use data, high loss to follow-up, and/or nonsystematic ascertainment of alcohol (ETOH) intake. We sought to systematically characterize ETOH use among WLS patients before surgery.
\end{abstract}

Methods-We recruited patients seeking WLS from May 2008 through November 2010 from two bariatric centers in Boston (response rate $75 \%$ ). Alcohol intake and problem-drinking behavior was systematically assessed in detail via phone interview among 653 obese patients before WLS. We used multivariable models to characterize alcohol drinking patterns and to examine the relationship between problem drinking and the likelihood of proceeding with WLS.

Results-At baseline, $29 \%$ were nondrinkers, $55 \%$ were social drinkers, and $16 \%$ were problem drinkers, including $4 \%$ who displayed alcohol abuse behavior. After adjustment, men and younger adults were significantly more likely to be high-risk drinkers. Problem drinkers were as likely as social drinkers to proceed with WLS.

Conclusion-Results from this large representative study suggest a high prevalence of high-risk alcohol use behavior among patients seeking WLS. Detailed assessment of alcohol use may be warranted in clinical settings and in studies of alcohol use and WLS.

\section{Keywords}

Alcohol abuse; Weight loss; Bariatric surgery; Preoperative; Outcomes

Obesity has risen rapidly in the US, with more than two thirds of adults having a body mass index $>25 \mathrm{~kg} / \mathrm{m} 2$ [1]. Weight-loss surgery has emerged as the most effective approach of ensuring substantial and sustained weight loss for many patients with severe obesity.

Presented as an oral presentation at the SAGES 2012 annual meeting, San Diego, CA, March 7-10, 2012.

Disclosures

O. Y. Kudsi, K. Huskey, S. Grove, G. Blackburn, D. B. Jones, and C. C. Wee have no conflicts of interest or financial ties to disclose. 
Though the majority of weight-loss surgery patients achieve a $>50 \%$ excess weight loss (EWL), there is a significant minority (15-20\%) who fail to achieve this goal [2, 3]. Failure to achieve successful weight loss after surgery is most likely multifactorial. Moreover, weight-loss surgery is not without its risk. Increasing emphasis is placed on identifying patient behavioral factors that correlate with successful weight loss and adverse effects.

While the prevalence of alcohol use and abuse among the US population is well documented [4-7], little is known about drinking patterns among those seeking WLS. It has been hypothesized that alcohol metabolism is altered after weight-loss surgery (WLS), with a few studies suggesting a high prevalence of postoperative problem drinking [8-12]. With the exception of the recently published data from the Longitudinal Assessment of Bariatric Surgery Study [11], these earlier studies were methodologically limited by lack of preoperative alcohol use data, high loss to follow-up, and/or nonsystematic ascertainment of alcohol (ETOH) intake. Hence, it is unclear whether reports of high-risk ETOH drinking behavior were truly unprecedented as opposed to preexisting behavior. We sought to systematically characterize ETOH use among patients seeking WLS and examine whether having high-risk ETOH drinking behavior was a factor in determining whether patients proceeded with WLS.

\section{Materials and methods}

The Assessment of Bariatric Surgery (ABS) Study [11] is a longitudinal cohort study of patients who were being evaluated for WLS. The primary goal of the study was to understand patient preferences and decision-making in the context of weight loss and WLS. Study subjects were systematically recruited from the WLS centers of two academic medical centers in Boston, one of which serves a large racial minority and socially disadvantaged urban population. To be eligible, patients had to be 18-65 years old at the time of recruitment, speak English, and have the permission of their physician for us to contact the patient. Patients were recruited from May 2008 through November 2010. The study was approved by the institutional review boards (IRB) at Beth Israel Deaconess Medical Center and Boston Medical Center.

Patients were administered a $1 \mathrm{~h}$ long telephone interview at baseline with an overall response rate of $75 \%(n=654)$. In addition to demographic and clinical factors, we assessed alcohol intake and high-risk drinking behavior using adapted items from the Alcohol Use Disorders Identification Test (AUDIT) instrument [13, 14]. The items used to assess alcohol intake are included in the Appendix. To ensure that patients would be candid with their response, we assured patients that their responses would be kept confidential and not reported back to their respective Bariatric Surgery programs. We classified patients' drinking patterns into several categories based on their responses to the first two questions about their frequency and quantity of alcohol intake within the last year. We first classified patients into either "never" drinkers (responded "never" to having a drinking containing alcohol in the past year) or current drinkers. Among men who were current drinkers in the past year, we categorized them as heavy drinkers if they reported having on average 3-4 drinks a day at least four or more times a week or 5-6 drinks a day at least two or more days a week. Women were considered heavy drinkers if they reported having on average at least 3-4 drinks on at least two more days a week. Current drinkers who did not meet criteria for heavy drinking were classified as social drinkers. We then asked whether patients drank 5 or more drinks within a day in the past month (i.e., evidence of binge drinking). Among patients who reported more than 1-2 drinks on a typical day or who reported at least one episode of binge drinking in the previous month, we then also asked a series three items related to alcohol dependency (impaired control over drinking, increased salience of drinking, and morning drinking) and four items related to harmful alcohol use (guilt after 
drinking, blackouts, alcohol-related injury, others concerned about drinking) (see Appendix). Anyone who responded affirmatively to questions pertaining to alcohol dependency or alcohol-related harm was defined as an alcohol abuser. We defined problem drinking based on those who met the criteria for heavy or binge drinking or who met the criteria for alcohol abuse.

We used descriptive statistics to characterize drinking patterns across different demographic factors. To examine demographic factors associated with exhibiting problem-drinking behavior, we developed a logistic regression that included age, sex, BMI, race/ethnicity, and surgery site. Further adjustments for education and income did not significantly change results and are not reported. We then developed a multivariable logistic regression model to examine whether the alcohol drinking pattern was associated with proceeding with WLS; the model was adjusted for potential confounders such as age, sex, BMI, race, surgery site, level of education, and income. All statistical analyses were conducted using SAS (SAS Institute, Cary, NC).

\section{Results}

The study cohort was mostly female (75\%). The cohort had a mean age of 44 years and a mean BMI of $46.5 \mathrm{~kg} / \mathrm{m} 2$, and $34 \%$ of the patients were non-white. At baseline, $29 \%$ were nondrinkers, $55 \%$ were social drinkers, $16 \%$ were problem drinkers, and $4 \%$ exhibited patterns consistent with alcohol abuse.

Table 1 presents the demographic correlates of high-risk drinking in our sample before adjustment for potential confounders. Men and racial and ethnic minorities were more likely than women and Caucasian patients, respectively, to exhibit problem drinking; however, differences were statistically significant across race/ethnicity but not by sex (borderline, $p<$ 0.1 ). In contrast, older adults were significantly less likely to exhibit problem drinking. After adjustment, sex and age remained statistically significant correlates of high-risk drinking (Table 2) but race and ethnicity were not longer important. The adjusted odds ratio was 1.98 $(95 \% \mathrm{CI}=1.22-3.22)$ for men compared to women and $0.62(0.50-0.77)$ for every higher decade in age. Interestingly, while never drinkers were significantly less likely to proceed with WLS, problem drinkers were as likely as social drinkers to proceed with WLS after adjustment for potential confounders (Table 2).

\section{Discussion}

In our sample of over 650 patients seeking weight-loss surgery (WLS), we found that a substantial minority of patients (16\%) exhibited problem alcohol drinking behavior. Moreover, patients who were problem drinkers were no less likely to proceed with WLS. Men seeking WLS were more likely than women seeking WLS to exhibit problem drinking whereas older adults were much less likely than younger adults.

There is emerging interest in understanding the risks for alcohol abuse among WLS patients. A recent national study reported that $15.4 \%$ of surgeons met the diagnostic criteria for alcohol abuse or dependence [15]. Alcohol abuse is very important with respect to WLS because of addiction concerns since there is increased sensitivity to alcohol due to digestive alterations that promote augmented absorption of alcohol. In addition, successful weight loss may not be achieved with alcohol abuse due to the high caloric composition of alcohol. A second concern arises from the concept of addiction transfer, where food may be replaced by another substance as a coping strategy. A subset of patients who undergo WLS may more apt to substitute their reliance on food as a coping strategy to abusing alcohol. Given the 
addiction concerns, we advise our patients to be extremely cautious and generally avoid alcohol $[14,15]$.

Multiple reports raised concerns that patients may be at an increased risk for developing an alcohol use disorder following WLS [12, 16-19]. However, most studies either had a small sample size, had very low retention rates, or, more importantly, did not assess alcohol use at baseline; thus, it is unclear whether disordered alcohol use after WLS was a new condition as opposed to preexisting. Suzuki et al. [17] assessed patients who underwent WLS with axis I disorders using the Structured Clinical Interview for DSM-IV and reported on 51 patients (11.1\% response rate); assessments were not conducted prior to surgery. They reported a lifetime prevalence of alcohol use disorder of $35 \%$ and reported a current prevalence of $11.8 \%$. Although they found no association between weight loss and current disordered drinking, their study was underpowered to address this question conclusively. Results from the Longitudinal Assessment of Bariatric Surgery Study suggest that the risk of alcohol use disorder does not occur until 2 years after WLS and the risk appears to be small [12]. Interestingly, all instances of current alcohol use disorder were identified in patients undergoing Roux-en-Y gastric bypass as opposed to laparoscopic adjustable gastric banding (LAGB) $[12,17]$. Our finding that a large minority of patients exhibit high-risk alcohol drinking behavior at baseline points to the importance of adequately assessing alcohol drinking patterns prior to WLS using studies aimed as determining whether WLS has a detrimental effect on alcohol use. While both WLS clinical centers from which our patients were recruited conduct psychiatric and psychological assessments of substance abuse, including alcohol abuse, prior to surgery, our study found that patients who met our criteria for "problem drinking" were no less likely to proceed with WLS.

Because most studies on WLS do not assess alcohol use systematically, there are insufficient data to suggest that patients with preoperative alcohol abuse are likely to achieve comparable weight loss after WLS [18, 19]. Odom et al. [18] recently demonstrated that weight regain after Roux-en-Y gastric bypass is associated with postoperative concerns about alcohol and substance abuse. In contrast, Dixon et al. [19] demonstrated that alcohol intake was actually associated with greater weight loss 1 year post-WLS.

Our findings suggest that problem drinking among patients seeking WLS is consistent with some of the prevalence data reported for the general population in the US. Hasin et al. [20] found that prevalence of lifetime and 12 month alcohol abuse was 17.8 and $4.7 \%$, respectively; prevalence of lifetime and 12 month alcohol dependence was 12.5 and $3.8 \%$, respectively. Alcohol dependence and abuse were significantly more prevalent among men, whites, Native Americans, and younger adults. Our prevalence of heavy drinkers is also consistent with the prevalence noted among patients seeking WLS in the Longitudinal Assessment of Bariatric Surgery Study [12]. Given the high prevalence of disordered and high-risk alcohol use in our study and in the general population, more attention should be paid to preoperative alcohol use.

Our study should be interpreted in the context of its limitations. Our data are based on selfreport and there may have been an element of respondent bias where patients report what they think is desirable. To minimize this, we assured patients that their responses would be confidential and not reported to the clinical team. Our results reflect those of patients seen at two WLS centers who participated in our study (although our response rate was high given the length of our interview). Future studies are needed to confirm our findings. Finally, several methods exist for assessing alcohol use and our results must be interpreted in that context [12, 16-20]. 
In summary, in this large study of patients seeking WLS, a substantial minority of patients exhibited problem-drinking behavior. Moreover, many of these patients proceeded with surgery, suggesting that detailed assessments of alcohol use are necessary in clinical settings. Future studies are needed to examine the interrelationships among alcohol use, WLS, and the outcomes of WLS.

\section{Supplementary Material}

Refer to Web version on PubMed Central for supplementary material.

\section{Acknowledgments}

The study was funded by a grant (to PIW) from the National Institutes of Diabetes and Digestive Diseases (R01 DK073302).

\section{References}

1. Flegal KM, et al. Prevalence and trends in obesity among US adults, 1999-2008. JAMA. 2010; 303(3):235-241. [PubMed: 20071471]

2. O'Brien PE, et al. Systematic review of medium-term weight loss after bariatric operations. Obes Surg. 2006; 16(8):1032-1040. [PubMed: 16901357]

3. Suter M, et al. Laparoscopic Roux-en-Y gastric bypass: significant long-term weight loss, improvement of obesity-related comorbidities and quality of life. Ann Surg. 2011; 254(2):267-273. [PubMed: 21772127]

4. Substance Abuse and Mental Health Services Administration. Results from the 2008 National Survey on Drug Use and Health: National Findings. 2009Office of Applied StudiesRockville Substance NSDUH Series H-36, HHS Publication No. SMA 09-4434

5. Grant BF, Dawson DA, Stinson FS, et al. The 12-month prevalence and trends in DSM-IV alcohol abuse and dependence: United States, 1991-1992 and 2001-2002. Drug Alcohol Depend. 2004; 74:223-234. [PubMed: 15194200]

6. National Institutes of Health. [19 Feb 2012] Alcohol use and alcohol use disorders in the United States: a 3-year follow-up. Main findings from the 2004-2005 wave 2 National Epidemiologic Survey on Alcohol and Related Conditions (NESARC). U.S. Alcohol Epidemiologic Data Reference Manual, vol 8, Number 2. 2010. http://pubs.niaaa.nih.gov/publications/ NESARC_DRM2/NESARC2DRM.pdf.

7. Narrow WE, Rae DS, Robins LN, Regier DA. Revised prevalence estimates of mental disorders in the United States: using a clinical significance criterion to reconcile 2 surveys' estimates. Arch Gen Psychiatry. 2002; 59(2):115-123. [PubMed: 11825131]

8. Hagedorn JC, Encarnacion B, Brat GA, et al. Does gastric bypass alter alcohol metabolism? Surg Obes Relat Dis. 2007; 3(5):543-548. [PubMed: 17903777]

9. Maluenda F, Csendes A, De Aretxabala X, et al. Alcohol absorption modification after laparoscopic sleeve gastrectomy due to obesity. Obes Surg. 2010; 20:744-748. [PubMed: 20358306]

10. Klockhoff H, Naeslund I, Jones AW. Faster absorption of ethanol and higher peak concentration in women after gastric bypass surgery. Br J Clin Pharmacol. 2002; 54:587-591. [PubMed: 12492605]

11. Ertelt TW, Mitchell JE, Lancaster K, et al. Alcohol abuse and dependence before and after bariatric surgery: a review of the literature and report of a new data set. Surg Obes Relat Dis. 2008; 4:647650. [PubMed: 18420465]

12. King WC, et al. Prevalence of alcohol use disorders before and after bariatric surgery. JAMA. 2012; 307:2516-2525. [PubMed: 22710289]

13. Babor, TF., et al. The alcohol use disorders identification test. Guidelines for Use in Primary Care. 2nd edn.. Department of Mental Health and Substance Abuse, World Health Organization; 2001. http://whqlibdoc.who.int/hq/2001/WHO_MSD_MSB_01.6a.pdf. 
14. Bush K, Kivlahan DR, McDonell MB, et al. Ambulatory Care Quality Improvement Project (ACQIP). The AUDIT alcohol consumption questions (AUDIT-C): an effective brief screening test for problem drinking. Arch Intern Med. 1998; 158(16):1789-1795. [PubMed: 9738608]

15. Oreskovich MR, Kaups KL, Balch CM, et al. Prevalence of alcohol use disorders among American surgeons. Arch Surg. 2012; 147(2):168-174. [PubMed: 22351913]

16. Sogg S. Alcohol misuse after bariatric surgery: epiphenomenon or "Oprah" phenomenon? Surg Obes Relat Dis. 2006; 3:366-368. [PubMed: 17452022]

17. Suzuki J, Haimovici F, Chang G. Alcohol use disorders after bariatric surgery. Obes Surg. 2012; 22:201-207. [PubMed: 21188544]

18. Odom J, Zalesin K, Tamika L, et al. Behavioral predictors of weight regain after bariatric surgery. Obes Surg. 2010; 20:349-356. [PubMed: 19554382]

19. Dixon JB, Dixon ME, O'Brien PE. Pre-operative predictors of weight loss at 1-year after Lap-Band surgery. Obes Surg. 2001; 11:200-207. [PubMed: 11355027]

20. Hasin DS, Stinson FS, Ogburn E, et al. Prevalence, correlates, disability, and comorbidity of DSMIV alcohol abuse and dependence in the United States. Arch Gen Psychiatry. 2007; 64(7):830-842. [PubMed: 17606817] 


\section{Table 1}

Pattern of alcohol use among weight-loss surgery patients at baseline

\begin{tabular}{lllll}
\hline & Nondrinkers $(\%)$ & Social drinkers $(\%)$ & High-risk drinkers $(\%)$ & $\begin{array}{l}\chi^{2} \text { test for difference in } \\
\text { proportions by sex, age, and race }\end{array}$ \\
Overall $(n=653)$ & $29(n=189)$ & $55(n=356)$ & $16(n=106)$ & \\
Men $(n=161)$ & $30(n=49)$ & $48(n=77)$ & $22(n=35)$ & $p<0.10$ \\
Women $(n=490)$ & $29(n=140)$ & $57(n=279)$ & $14(n=71)$ & \\
Age 18-29 years $(n=100)$ & $28(n=28)$ & $45(n=45)$ & $27(n=27)$ & $p<0.0001$ \\
Age 30-44 years $(n=223)$ & $23(n=50)$ & $57(n=127)$ & $20(n=45)$ & \\
Age 45+ years $(n=330)$ & $34(n=111)$ & $56(n=184)$ & $10(n=34)$ & \\
Non-Hispanic white $(n=430)$ & $26(n=110)$ & $60(n=256)$ & $15(n=64)$ & \\
Non-Hispanic AA $(n=115)$ & $31(n=36)$ & $50(n=58)$ & $18(n=21)$ & \\
Hispanic and other $(n=105)$ & $40(n=42)$ & $40(n=42)$ & $20(n=21)$ & \\
\hline
\end{tabular}




\section{Table 2}

Association between pattern of alcohol use at baseline and likelihood of proceeding with weight-loss surgery

\begin{tabular}{llll}
\hline & Proceeding w/WLS $(\%)$ & Odds ratio & 95 \% CI \\
Nondrinkers & 76 & 0.67 & $0.41-1.09$ \\
Social drinkers & 87 & 1.00 & Reference \\
Problem drinkers & 83 & 0.93 & $0.49-1.74$ \\
\hline
\end{tabular}

Model adjusted for age, BMI, sex, race/ethnicity, recruitment site, education, and income 